Wolfgang H. Lorig

\title{
Verantwortliche Amtsführung im demokratischen Verfassungsstaat
}

\section{Korruption als aktuelle Herausforderung}

Der Korruptionsbericht der OECD und entsprechende Studien der Weltbank erweitern den Rahmen für eine wissenschaftliche Auseinandersetzung mit dem Thema »Amtsgedanke«, »Ämterordnung « und »verantwortliche Amtsführung « ${ }^{1}$ : Denn Korruption setzt den Missbrauch eines öffentlichen Amtes voraus²; erst der Missbrauch eines öffentlichen Amtes durch den oder die Amtsinhaber macht Korruption möglich. Ist dieser Zusammenhang zutreffend, dann schwindet in der Bundesrepublik bei den Amtsträgern das Verantwortungsbewusstsein für das öffentliche Amt. In der internationalen Korruptionsstatistik von Transparency International befindet sich die Bundesrepublik seit 1995 auf dem Weg ins untere Mittelfeld und die Statistik der Strafverfolgung von Straftaten im Amt belegt seit 1993 einen deutlichen Anstieg der Verurteilungen wegen Bestechlichkeit und Vorteilnahme ${ }^{3}$. Korruption scheint inzwischen »in Deutschland in der Müllwirtschaft, genauso wie in der Baubranche oder in der Medizintechnik, zur Unternehmenskultur « ${ }^{4}$ zu gehören. Allerdings wird dort, wo Korruption geduldet ist, sich diese auch ausbreiten. Und wo diese sich ausbreiten kann, wird der demokratische Verfassungsstaat schnell an Boden verlieren5.

1 OECD, No Longer Business as Usual. Fighting against Bribery and Corruptions, Paris 2000, Einband: »Today, corruption has moved to the top of the global political agenda as its dramatic impact on economic development and its corrosive effect on political stability and democratic political institutions has become increasingly obvious. « Siehe auch Susan RoseAckerman, Corruption and Development. Paper prepared for the Annual World Bank Conference on Development Economics, Washington, D. C., April 30 and May 1, 1997.

2 Vgl. »Bribery. The worm that never dies« in: The Economist, 2. - 8. März 2002, S. 11.

3 Vgl. u. a. »Deutschland rutscht in der Korruptionsliste ab « in: FAZ, Nr. 147, 28. Juni 2001, S. 15; Christoph Seidler, Korruptions-Rangliste: Deutschland schneidet schlecht ab. Internetdokument: http://www.spiegel.de/politik/deutschland/0,1518,142022,00.html (10.07.2001); Peter Eigen, Das Netz der Korruption, Frankfurt a. M. 2003.

4 Matthias Krupa, »Made in Germany. Korruption zerfrisst den Staat, die Parteien fressen mit « in: Die Zeit, 18. April 2002, S. 1. Ebenso Wolfgang Schaupensteiner, zitiert in: FAZ, Nr. 252, 30. Oktober 2001, S. 3. Vgl. auch Hans Leyendecker, Die Korruptionsfalle, Reinbek 2003.

5 In diesem Sinne: Heinrich Oberreuter, »Politik und Moral« in: Günter Tondorf (Hg.), Staatsdienst und Ethik. Korruption in Deutschland, Baden-Baden 1995, S. 47 ff., bes. S. 49 f., 53 f. Siehe auch: Angela Drösser, Korruption in Deutschland: Ursachen, Erscheinungsformen, Bekämpfungsstrategien, Berlin 1995; Detlef Krauss, Korruption in Deutschland, Berlin 1996. 
In Kenntnis dieser Zusammenhänge re-thematisieren die OECD, die Weltbank und verschiedene Nachbardisziplinen der Politikwissenschaft mit ihren Überlegungen zu Good Governance auch die normative Dimension eines guten Regierens und Verwaltens $^{6}$ : Karl-Peter Sommermann fragt, ob wir »eine Ethik des öffentlichen Dienstes « und die Ausbildung eines »belastbaren Amtsethos « ${ }^{7}$ brauchen, Peter Ulrich erörtert die Bedeutung einer Reformulierung der klassischen Einsichten einer republikanischen Ethik angesichts einer institutionell entfesselten und normativ enthemmten ökonomischen Rationalität, die »real die fortschreitende, tendenziell grenzenlose Ökonomisierung aller Lebensbereiche « ${ }^{8}$ betreibt. Klaus König9 reflektiert die Zusammenhänge zwischen Steuerungs- und Wertefragen, und Elke Löffler erörtert die Interdependenzen zwischen Korruptionsbekämpfung und der aktuellen Ethikdiskussion ${ }^{10}$.

Auch wenn »die Ambivalenz im Normativen in einem gewissen Sinn fester Bestandteil $\ll^{11}$ von Korruption ist, geht es bei diesem Problem im Kern um die Analyse der Zusammenhänge zwischen einem wertgebundenen Amtsverständnis, der Integrität der Amtsinhaber und den strukturell-institutionellen Komponenten einer freiheitlich-demokratischen Verfassungsordnung, somit um die Prinzipien und Rahmenbedingungen eines »guten Regierens « ${ }^{12}$.

\section{Amtsmissbrauch und Korruption}

Das Korruptionsproblem ist vielschichtig, und der Korruptionsbegriff variiert nach Kulturkreis, historischer Situation und politischem bzw. ökonomischem System.

6 Klaus König, »Good Governance - as Steering and Value Concept for the Modern Administrative State in: Joan Corkery (Hg.), Gouvernance: Concepts et Applications, Bruxelles 1999, S. 67 ff.; Adam Markus, »Die Entstehung des Governance-Konzepts bei

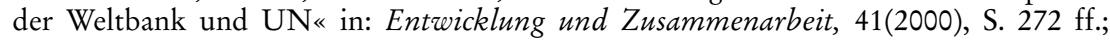
Christian Theobald u. a., »Governance als Wertekonzept« in: Klaus König u. a., Governance als entwicklungs- und transformationspolitisches Konzept, Berlin 2002, S. $277 \mathrm{ff}$.

7 Karl-Peter Sommermann, »Brauchen wir eine Ethik des öffentlichen Dienstes? in: Verwaltungsarchiv, 89 (1998), S. 290 ff., S. 290. Siehe auch Kathryn G. Denhardt, The Ethics of Public Service, New York u. a. 1988.

8 Peter Ulrich, Integrative Wirtschaftsethik als kritische Institutionenethik. Wider die normative Überhöbung der Sachzwänge des Wirtschaftssystems. Beiträge und Berichte Nr. 62 der Hochschule St. Gallen, St. Gallen 1994, S. 4.

9 Klaus König, »Zur Steuerungs- und Wertproblematik « in: ders. / Markus Adam (Hg.), Governance als entwicklungspolitischer Ansatz. Speyerer Forschungsberichte 219, Speyer 2001, S. $189 \mathrm{ff}$.

10 Elke Löffler, »Ethikdiskussion und Korruptionsbekämpfung im öffentlichen Sektor der OECD-Mitgliedsländer « in: Albert Hofmeister (Hg.), Brauchen wir eine neue Ethik in der Verwaltung?, Bern 2000, S. $141 \mathrm{ff}$.

11 Vgl. Christian Fleck / Helmut Kuzmics, Einleitung in: dies. (Hg.), Korruption. Zur Soziologie nicht immer abweichenden Verhaltens, Königstein/Ts. 1985, S. 3 ff., S. 30.

12 Vgl. Ernst Vollrath, »Überlegungen zur Semantik von >Regierung< und >Regieren« in: Hans-Hermann Hartwich / Göttrik Wewer (Hg.), Regieren in der Bundesrepublik 1. Konzeptionelle Grundlagen und Perspektiven der Forschung, Opladen 1990, S. 65 ff. 
Eine eindeutige wissenschaftliche Begriffsdefinition, die länder-, zeit- und systemübergreifend wäre, liegt nicht vor. Mit Thomas Heberer lassen sich nachstehende Kriterien für korrupte Tätigkeit aufzählen:

- »Es muss ein Funktionsträger (oder ein kurzfristig mit Funktionen Beauftragter) bzw. eine Gruppe von Funktionsträgern im nicht-privaten Sektor involviert sein. (...)

- Es müssen staatlich gesetzte Normen (...) ethisch-moralische und/oder gesellschaftliche oder politische Standards verletzt werden, wobei auch die Normverletzung durch die politische Elite (...) einbegriffen sein muss.

- Durch die Amtsverletzung erhält der Funktionsträger einen Vorteil oder Anreiz, der nicht pekuniär zu sein braucht.

- Durch die Amtsverletzung nimmt der Funktionsträger eine begünstigende Handlung für eine entlohnende Seite vor bzw. unterlässt eine nachteilige. « ${ }^{13}$

Bestandteile dessen, was die Sozial- und Wirtschaftswissenschaftler allgemein als Korruption bezeichnen, finden wir als Einzeltatbestände in unseren Rechtskatalogen: Vorteilsnahme, Bestechlichkeit, Vorteilsgewährung und Bestechung, aber auch Ämterpatronage $\mathrm{e}^{14}$ und Bestechlichkeit von Abgeordneten ( $\left.\mathbb{S} 108 \mathrm{e} \mathrm{StGB}\right) »$ beim konkret nachgewiesenen Stimmenkauf (...) vor einer Abstimmung « ${ }^{15}$.

Primär geht es hierbei um Straftaten im Amt, die durch eine »Verletzung des Treueverhältnisses zum Staat oder des Vertrauens der Öffentlichkeit in die Integrität des Beamtenapparates gekennzeichnet sind ${ }^{16}$. Das rechtliche Normensystem kennt Korruption als Amtspflichtverletzung erst seit der Herausbildung eines Verwaltungs- und Beamtenapparates, der sich als »Diener des Staates « versteht. Max Weber hat das Modell sachlicher Amtspflicht des deutschen Beamten als Idealtypus rationaler legaler Herrschaft der Bürokratie beschrieben: »Ihr Ideal ist, ssine ira et studio<, ohne allen Einfluss persönlicher Motive oder gefühlsmäßiger Einflüsse, frei von Willkür und Unberechenbarkeiten, insbesondere sohne Ansehen der Person< streng formalistisch nach rationalen Regeln und - wo diese versagen - nach sachlichen<Zweckmäßigkeitsgesichtspunkten zu verfügen. «17

Ganz im Sinne Max Webers imponiert das normative Programm des bundesdeutschen Beamtenrechts durch Konsequenz und Geschlossenheit. Relativ unbeirrt durch moderne Pflichtenallergie bewahren die Beamtengesetze (noch) die Semantik der traditionellen Pflichten. Formulierungen wie: der Beamte »hat sein Amt unei-

13 Thomas Heberer, Politische Korruption in China. Analyse eines politischen, ökonomischen und sozialen Problems, Opladen 1991, S. 21 f.

14 Vgl. Jürgen Bellers / K. Schöler, »In der Grauzone von Recht und Politik « in: Jürgen Bellers (Hg.), Politische Korruption in der Bundesrepublik, Münster 1989, S. 82 ff.

15 »Immer untendurch. Bei Korruption gelten für Abgeordnete bis heute Sonderregeln « in: Der Spiegel, 2002, Nr. 14, S. 44 ff., S. 44. Siehe auch »Die Vettern der Wirtschaft« in: Der Spiegel, 2003, Nr. 26, S. 36 ff.

16 Adolf Schönke / Horst Schröder / Walter Penon / Günter Heine, Strafgesetzbuch, Kommentar, 26. neu bearbeitete Aufl. München 2001, S. 2568, Rn. 1.

17 Max Weber, »Die drei reinen Typen der legitimen Herrschaft« in: ders., Weltgeschichtliche Analysen, Politik, hg. von Johannes Winckelmann, Stuttgart 1964, S. 152. 
gennützig nach bestem Gewissen zu verwalten $\aleph^{18}$, er »dient dem ganzen Volk, nicht einer Partei« oder er hat sich » mit voller Hingabe seinem Beruf zu widmen«, wollen verdeutlichen, dass »Amts- und Dienstpflichten sich nicht vollständig verrechtlichen lassen, dass ein überschießender ethischer Rest bleibt, mehr noch, dass das Amt des Beamten wie jedes andere Amt auch ethische Erwartungen an seinen Inhaber verkörpert «19.

Der konsequenten Regelungsdichte im administrativen Bereich entspricht keine vergleichbare Verrechtlichung im gubernativen Bereich. Das »Gesetz über die Rechtsverhältnisse der Mitglieder der Bundesregierung « enthält in $\ 5$ Abs. 3 des »Bundesministergesetzes « die Regelung, dass »die Mitglieder und ehemaligen Mitglieder der Bundesregierung (...) dieser über Geschenke Mitteilung zu machen (haben), die sie in bezug auf ihr Amt erhalten«. Doch gilt gemäß $\$ 8$ desselben Gesetzes, dass »ein Verfahren gegen Mitglieder der Bundesregierung wegen eines etwaigen Verstoßes gegen die Mitteilungspflicht (...) nicht statt(findet)«. Korruptheit ist durchaus ein Verstoß gegen die im Amtseid ${ }^{20}$ beschworenen Pflichten eines Regierungsmitglieds. Vergegenwärtigt man sich das gültige amtsrechtliche Regelungsprogramm, fällt sie aber nicht in die rechtliche, sondern »nur « in die politische Verantwortlichkeit des Regierungsmitglieds ${ }^{21}$.

Die angedeutete fehlende bzw. geringe Verrechtlichung im gubernativen Bereich wird indes durch das Strafrecht relativiert: Gemäß $\ 11$ Abs. 1 Nr. 2 StGB sind Amtsträger nicht nur Beamte im staats- und verwaltungsrechtlichen Sinne, sondern auch diejenigen Personen, die »in einem sonstigen öffentlich-rechtlichen Amtsverhältnis «22 stehen. Strafrechtlich wird zur Verantwortung gezogen, wer als Amtsträger einen Vorteil als Gegenleistung dafür fordert, sich versprechen lässt oder annimmt, dass er eine Diensthandlung vorgenommen hat oder künftig vornehme. In den $\mathbb{S}$ 331-334 spricht das Strafgesetzbuch von Amtsträgern ${ }^{23}$ bzw. von für den öffentlichen Dienst besonders Verpflichteten und differenziert dann auf der Passivsei-

$18 \int 36$ Beamtenrechtsrahmengesetz. Vgl. auch die Leitentscheidung des BGH, BGHSt, 15, S. 88 ff., S. 96 f: »Die Makellosigkeit des Amtes nach außen soll gesichert werden; mit der Ehrenhaftigkeit und vor allem Unbestechlichkeit des Beamten soll die Grundlage für das Vertrauen der Bevölkerung erhalten werden, dessen die Staatsverwaltung für eine gedeihliche Wirksamkeit bedarf (...).《

19 Josef Isensee, »Das Amt als Medium des Gemeinwohls in der freiheitlichen Demokratie« in: Gunnar Folke Schuppert / Friedhelm Neidhardt (Hg.), Gemeinwobl-Auf der Suche nach Substanz. WZB-Jabrbuch 2002, Berlin 2002, S. 241 ff., S. 263.

20 Vgl. Art. 64, 2 und Art 56 GG i. V. mit $\$ 3$ Ministergesetz.

21 Vgl. Klaus Kröger, Die Ministerverantwortlichkeit in der Verfassungsordnung der Bundesrepublik Deutschland, Frankfurt a. M. 1972, S. 12 ff.

22 Amtsträger ist nach der Legaldefinition des $\ 11$ Abs. 1 Nr. 2 StGB, "wer nach deutschem Recht a) Beamter oder Richter ist, b) in einem sonstigen öffentlich-rechtlichen Amtsverhältnis steht oder c) sonst dazu bestellt ist, bei einer Behörde oder einer sonstigen Stelle oder in deren Auftrag Aufgaben der öffentlichen Verwaltung wahrzunehmen $\ll$.

23 Zum Amtsträgerbegriff: Jürgen Welp, »Der Amtsträgerbegriff« in: Wilfried Küper (Hg.), Festschrift Karl Lackner zum 70. Geburtstag am 18. Februar 1987, Berlin/New York 1987, S. $761 \mathrm{ff}$. 
te zwischen Vorteilsnahme und Bestechlichkeit sowie auf der Aktivseite zwischen Vorteilsgewährung und Bestechung ${ }^{24}$.

Anders als bei den Regierungsmitgliedern sind die Abgeordnetenpflichten, was die Annahme von Zuwendungen angeht, ausdrücklich normiert. Gemäß $\$ 9$ Abs. 1 GOBT, der die Verhaltensregeln für die Mitglieder des Deutschen Bundestages ausführt und auf $\ 44$ a des Abgeordnetengesetzes basiert, darf »ein Mitglied des Bundestages (...) in Ausübung des Mandats keine anderen als die gesetzlich vorgesehenen Zuwendungen oder andere Vermögensvorteile annehmen «. Und $\ 44$ a Abs. 2, 4 Abgeordnetengesetz spricht von der "Unzulässigkeit einer Annahme von Zuwendungen, die das Mitglied des Bundestages, ohne die danach geschuldeten Dienste zu leisten, nur deshalb erhält, weil von ihm in Hinblick auf sein Mandat erwartet wird, dass es im Bundestag die Interessen des Zahlenden vertreten und nach Möglichkeit durchsetzen wird «.

Doch die Inkorporation des Straftatbestands der Abgeordnetenbestechung in das Strafgesetzbuch ( $\mathbb{1} 108 \mathrm{e}$ StGB) im Jahre 1994 pönalisiert nur den Stimmenkauf bzw. -verkauf bei Wahlen und Abstimmungen im Parlament. Der Abgeordnete wird anders als das Regierungsmitglied - nicht zum Amtsträger im strafrechtlichen Sin$\mathrm{ne}^{25}$. Das Gewähren von Zuwendungen durch interessierte Dritte an Abgeordnete bleibt strafrechtlich irrelevant, sieht man von dem seltenen Fall des Stimmenkaufs bzw. Stimmenverkaufs bei Wahlen und Abstimmungen im Parlament ab. Auch nach der Inkorporation des Straftatbestands der Abgeordnetenbestechung in das StGB wird - im Gegensatz zum Minister bzw. Regierungsmitglied - eine strafrechtliche Amtsträgerschaft des Abgeordneten allgemein verneint ${ }^{26}$, obwohl Art. 48 Abs. 2 S. 1 GG ausdrücklich vom Amt eines Abgeordneten spricht.

Beamteneigenschaft und die sonstigen öffentlich-rechtlichen Amtsverhältnisse ${ }^{27}$ haben ein gemeinsames sachliches Substrat: »die besondere personale Bindung der Amtsträger an den Staat ${ }^{28}$. Doch die Verrechtlichungsgrade des administrativen, des gubernativen und des repräsentativ-politischen Bereichs bleiben unterschiedlich. Insoweit verdeutlichen die skizzierten Gesetzes- und Verhaltensregeln, dass es der Bekämpfung von Korruption - insbesondere im nicht-administrativen Bereich - an

24 Vgl. Bernhard Haffke, »Politik und Korruption/Strafrechtliche Notizen zu den jüngsten Bestechungsskandalen « in: Günter Tondorf (Hg.), aaO. (FN 5)., S. 11 ff., S. 15.

25 Vgl. ebd., S. 22; Wolfgang Schaupensteiner / Christian Bommarius, »Filz und Speck « in: Kursbuch, »Korruption«, Juni 1995, H. 120, S. 36 ff., S. 40.

26 Vgl. Adolf Eser, »\$108e« in: Adolf Schönke / Horst Schröder / Walter Penon / Günter Heine (Hg.), aaO. (FN 16), Rn. 8 f.; Bernhard Haffke, aaO. (FN 24), S. 22; Hans-Ulrich Paeffgen, "Amtsträgerbegriff und Unabhängigkeit des Datenschutzbeauftragten « in: Juristenzeitung, 52 (1997), H. 4, S. 178 ff., S. 179 ff.; Hans-Joachim Rudolphi u. a., Systematischer Kommentar zum Strafgesetzbuch, Loseblattsammlung, Stand 2003, Bochum u. a. April 2003, $\mathbb{1 1}$, Rn. 17.

27 Vgl. BT-Drucksache 7/550, 1973, S. 209. Die Minister des Bundes und der Länder werden dort ausdrücklich als Beispielsfälle aufgeführt.

28 Jürgen Welp, aaO. (FN 23), S. 764. Ausführlich: Bernd Heinrich, Der Amtsträgerbegriff im Strafrecht. Auslegungsrichtlinien unter besonderer Berücksichtigung des Rechtsguts der Amtsdelikte, Berlin 2001. 
Normenklarheit nach wie vor mangelt. Die Politikwissenschaft tut deshalb gut daran, Korruption nicht allein über strafrechtliche Tatbestände wie Vorteilsnahme, Bestechlichkeit, Vorteilsgewährung und Bestechung, also die Aktionen oder unterlassenen Aktionen vor allem der Beamtenschaft $\mathrm{zu}$ definieren und $\mathrm{zu}$ diskutieren. Vielmehr sollte Korruption definiert werden »as a >deviation < from the public good. (...) For, in a democracy politicians become speople's representatives and bureaucrats spublic servants precisely because these functions deemed to be conducive to the common good. Corruption - simproper < behaviour - denotes deformation of this norm. It is the abuse of public office, for private gain, that constitutes corruption. $\ll^{29}$

In Übereinstimmung mit einem im Europarat im Jahre 1994 erörterten Begriffsverständnis kann Korruption als ein »Verhalten von Personen mit öffentlichen Aufgaben, die ihre Pflichten verletzen, um ungerechtfertigte Vorteile gleich welcher Art zu erhalten «30, definiert werden. Dieses Verständnis erfasst neben einem abweichenden Verhalten der Beamtenschaft auch das von der Öffentlichkeit als verwerflich wahrgenommene Verhalten von Regierungsmitgliedern und gewählten Repräsentanten, wenn diese öffentliche Ressourcen zweckentfremdend für private (oder politische) Vorteile nutzen, ohne dass ihnen unmittelbar ein strafrechtlich relevantes Handeln vorgeworfen werden kann ${ }^{31}$. »Korruption « in diesem weiten Sinne beinhaltet dann ganz allgemein die missbräuchliche Nutzung öffentlicher Ressourcen für private oder politische Zwecke, d. h. den Missbrauch eines öffentlichen Amtes $^{32}$.

29 John Girling, Corruption, Capitalism and Democracy, London/New York 1997, S. 2 f.

30 Vgl. Antwort der Niedersächsischen Landesregierung vom 26. August 1997 auf eine Große Anfrage der Fraktion der CDU, LT-Drucksache 13/3219, S. 4. Zur Problematik der Begriffsfassung siehe u. a.: Arnold J. Heidenheimer / Michael Johnston / Victor T. LeVine (Hg.), Political Corruption. A Handbook, New Brunswick/London 1997; Donatella Della Porta / Yves Mény (Hg.), Democracy and Corruption in Europe, London 1997.

31 Josef Isensee, aaO. (FN 19), S. 257 f.: »Mag das Bild des Amtes heute verblasst sein eine Amtspflicht ist im allgemeinen Rechtsbewusstsein lebendig geblieben: das Verbot, dass ein Amtsträger sich persönlich oder die Partei, der er angehört, aus öffentlichen Kassen finanziell bereichert. Hier geht es nicht allein um Bestechung im engen strafrechtlichen Verständnis, vielmehr um Korruption, die nicht tatbestandlich scharf abgrenzbar ist: um Amtsmissbrauch für eigennützige wirtschaftliche Zwecke. Das Verbot bezieht sich nicht nur auf Beamte, Richter und sonstige Amtsträger, die besonderen Straf- und Disziplinarvorschriften unterliegen. Es erfasst auch die Abgeordneten.«

32 So auch die Definition von Joseph S. Nye, »Corruption and Political Developments: A Cost-Benefit Analysis« in: Arnold J. Heidenheimer / Michael Johnston / Victor T. LeVine (Hg.), aaO. (FN 30), S. 963 ff., S. 966: Gemäß dem »Public-Office-Centered «Konzept soll (bürokratische) Korruption ein Verhalten sein, das abweicht von den »formal duties of a public role because of private-regarding (personal, close family, private clique) pecuniary or status gains; or violates rules against the exercise of certain types of private-regarding influence. This includes such behavior as bribery (use of reward to pervert the judgement of a person in a position of trust); nepotism (bestowal of patronage by reason of ascriptive relationship rather than merit); and misappropriation (illegal appropriation of public resources for private-regarding uses).« 


\section{Amtsgedanke und Ämterordnung}

Da der aktuelle Sprachgebrauch sinnvariabel ist, dürfte die Kenntnis der Wortgeschichte erhellend sein: Das keltische Wort ambactos, die lateinischen Termini ministerium, munus, officium, die althochdeutschen ampahti, ampaht sowie die frühneuhochdeutschen Begriffe ambt, ampt verweisen auf einen Dienst oder einen Dienenden $^{33}$. Die Wurzeln des modernen Amtsbegriffs lassen sich zurückverfolgen bis in die Behördenorganisation des Römischen Kaiserreichs. Während das öffentliche Amt in der frühen römischen Republik von einer "fiktiven Einheit von Amt und Amtsinhaber ausging ", vollzog sich vor allem während der Regierungszeit von Kaiser Diokletian »die strukturelle Trennung von Amt und Person « ${ }^{34}$. Der spätrömische, auf der Trennung von Amt und Person beruhende, funktionsbestimmte Amtsbegriff wurde zunächst von den germanischen Nachfolgestaaten beibehalten und lebte weiter sowohl im Fränkischen Reich als auch in der kirchlichen Kanzlei- und Verwaltungspraxis ${ }^{35}$. Die Auffassung vom officium ${ }^{36}$ als Tätigkeit und Ort amtlicher Durchführung dienstherrlich vorgegebener Ziele sowie später als Bezeichnung für das gesamte Büro und Personal eines Amtsträgers innerhalb des kaiserlichen Verwaltungssystems dient - in der kirchlichen Verwaltung des Mittelalters - der Kennzeichnung einer anvertrauten, zeitlich, sozial und sachlich befristeten Handlungsdelegation als Amtsgewalt in Abhebung zum lebenslänglichen, meist zudem vererbbaren beneficium. Widerrufbarkeit der Amtsübertragung und Absetzbarkeit durch fürstliche Entscheidung verhindern nicht nur die feudale erbliche Amtsnutzung, »sondern konstituieren eine neuartige Auffassung vom Amt als auftragsgebundener Handlungsdelegation aus dem Recht landesherrlicher Autorität $\ll^{37}$.

Seit dem 15./16. Jahrhundert beginnt der Amtmann der deutschen Territorien »öffentlich-rechtliche Funktionen kraft Auftrag ${ }^{38}$ auszuüben und kann sich dabei auf ein geschriebenes Organisationsrecht fürstlich erlassener Hof- und Ämterordnungen berufen ${ }^{39}$. Mit der strukturellen Trennung von Amt und Person entfaltet der funktionsbestimmte, sachliche Amtsbegriff weitere konstitutive Elemente: die handlungs- und kompetenztheoretische Teilbarkeit von Verantwortung und eine instanzliche Ämterhierarchie mit definierten Zuständigkeiten, Sachkompetenzen und

33 Vgl. Jacob und Wilhelm Grimm, Deutsches Wörterbuch, Bd. 1, Leipzig 1854.

34 Gerlinde Sommer, »Verantwortung als demokratisches Staatsprinzip « in: Raban Graf von Westphalen (Hg.), Deutsches Regierungssystem, München/Wien 2001, S. 99 ff., S. 104.

$35 \mathrm{Vgl}$. Joachim Ritter, Artikel »Amt« in: ders. (Hg.), Historisches Wörterbuch der Philosophie, Darmstadt 1971, Bd. 1, Sp. 210 ff.; Josef Isensee, aaO. (FN 19), S. 243-247.

36 Jacob und Wilhelm Grimm, aaO. (FN 33), Sp. 280 f. »Das jetzige amt drückt nun hauptsächlich den dienst, das geschäft aus, womit einer beauftragt ist (...).«

37 Gerlinde Sommer, Institutionelle Verantwortung. Grundlage einer Theorie politischer Institutionen, München/Wien 1997, S. 111.

38 Fritz Hartung, Deutsche Verfassungsgeschichte vom 15. Jahrbundert bis zur Gegenwart, Stuttgart 1969, S. 50.

39 Vgl. Wolfgang Sellert (Hg.), Die Ordnungen des Reichshofrates 1550-1766, 2 Halbbde., Köln 1980/1990. 
Handlungsbefugnissen ${ }^{40}$. Das aufscheinende Verständnis institutioneller Verantwortung ist dabei gekennzeichnet »durch die Auffassung vom Amt als erteilter Handlungszuständigkeit mit festen Kompetenzschranken« und durch eine »Souveränität, welche die enge Rückbindung an die autorisierende Instanz durch Rechenschaftslegung und Verantwortung zu garantieren hat ${ }^{41}$.

Im neuzeitlichen demokratischen Verfassungsstaat, den wir auch repräsentative Demokratie nennen ${ }^{42}$, tritt neben das Verwaltungsamt das politische Amt: Herrschaft als anvertrautes Amt auf Zeit. Herrschaft ist gemäß der Idee demokratischer Repräsentation in Ämtern verfasst und dadurch reguliert und diszipliniert. Ämter sind bezogen auf eine nicht eigennützige, sondern fremdnützige Wahrnehmung allgemeiner Angelegenheiten ${ }^{43}$. Als Institution besteht das Amt unabhängig vom personellen Amtsträger (Trennung von Amt und Person) und bestimmt sich maßgeblich nach den sachlich, sozial und zeitlich definierten Kompetenzen. Im Amt als institutionalisierter Form von Verantwortung werden Demokratie- und Amtsprinzip verknüpft. Mit seiner Ämterordnung und dem Moment des Wechselns in den Ämtern schafft der moderne Verfassungsstaat klare Verantwortungszeiten und -räume. Indem deutlich gemacht wird, wer sich in welchen zeitlichen Abständen vor welchen Instanzen und mit welchen Verfahren zu verantworten hat, "zerlegt« der demokratische Verfassungsstaat »die Machtausübung und macht sie dadurch der Übersicht und Kontrolle zugänglich $\aleph^{44}$. Ein Amt haben in dieser politischen Ordnung also nicht nur die Angehörigen der Exekutive und der Judikative inne, »sondern auch und sogar in erster Linie die unmittelbar vom Volk gewählten Mitglieder der Parlamente ${ }^{45}$. Wer ein öffentliches Amt inne hat, der muss sich vor der Gesamtheit der Bürger für das, was er mit seiner Vollmacht anfängt, auch verantworten können: »Verantwortung hat es wesentlich mit Fragen der Zuständigkeit, der Kompetenzen, der damit verbundenen Befugnisse und Pflichten von Amtsinhabern und deren Begründungs- und Rechenschaftspflicht gegenüber anderen zu tun. (...) Vertrauen und Verantwortung bilden in jedem freiheitlichen System (...) unverzichtbare Grundkategorien.«46

Konturen eines kompetenz- und amtsrechtlichen Verständnisses von Verantwortung zeichnet auch das Grundgesetz der Bundesrepublik Deutschland: Der Begriff

40 Vgl. Gerlinde Sommer, aaO. (FN 37), S. 115.

41 Ebd., S. 116.

42 Ausführlich: Ernst-Wolfgang Böckenförde, Staat, Nation, Europa, Frankfurt a. M. 1999, S. 127-140.

43 Ernst-Wolfgang Böckenförde, »Die Krise unserer Demokratie verlangt eine Rückbildung des Parteienstaates « in: FAZ, Nr. 37, 14. Februar 2000, S. 3.

44 Hans Maier, »Der Begriff der Politik« in: Zeitschrift für Politik, 34 (1987), H. 4, S. 378 ff., S. 381.

45 Rupert Hoffmann, »Demokratie zwischen Repräsentation und Anarchie« in: Zeitschrift für Politik, 31 (1984), H. 2, S. 123 ff., S. 129.

46 Winfried Steffani, »Vertrauen und Verantwortung in der Politik « in: Ingeborg Villinger / Gisela Riescher / Jürgen Rüland (Hg.), Politik und Verantwortung, Freiburg i. Br. 2000, S. 234 ff., S. 237 f. Zur Bedeutung von Vertrauen und Vertrauenskultur siehe Piotr Sztompka, Trust. A Sociological Theory, Cambridge 1999. 
Verantwortung findet sich - außer in der normativen Verwendung in der Präambel amtsrechtlich in den Art. 28 Abs. 2, 34, 42 Abs. 3, 46 Abs. 1 und 2 und Art. 65 GG. Es lassen sich wesentliche, dem römisch-rechtlichen Amtsbegriff zuzuordnende Strukturprinzipien konstatieren: u. a. eine zeitliche Befristung der Amtsinhabe, temporale wie örtliche Beständigkeit des Amtes, Ressort-, Kanzler- und Kabinettsprinzip. Der Teilbarkeit von Verantwortung folgend werden Kompetenzbereiche in alleiniger Zuständigkeit wahrgenommen; doch bei Amtspflichtverletzungen fällt die Verantwortung im Sinne einer institutionellen Verantwortung - unabweisbar auf die autorisierende Instanz zurück: »Verletzt jemand in Ausübung eines ihm anvertrauten öffentlichen Amtes die ihm einem Dritten gegenüber obliegende Amtspflicht, so trifft die Verantwortlichkeit grundsätzlich den Staat oder die Körperschaft, in deren Dienst er steht. « ${ }^{47}$

\section{Public Trust und Public Good im Responsible Government}

In den angelsächsischen Ländern zählen der Terminus responsible government und die Kategorie »politische Verantwortlichkeit « »um etablierten analytisch-kritischen Begriffsinstrumentarium der politischen Wissenschaft, der Verfassungsgeschichte und der öffentlichen Diskussion « ${ }^{48}$. Als eine bedeutende politiktheoretische Quelle für das Konzept verantwortlicher Regierung kann der Federalist bezeichnet werden ${ }^{49}$. Darin ist zwar nicht explizit eine Theorie des responsible government formuliert; doch die verfassungspolitischen Erwägungen entfalten ein Verständnis von demokratischer Ordnung, dem ein System verantwortlicher Regierung zugrundeliegt: Madisons Plädoyer für die Republik und gegen die »reine Demokratie« in den Briefen 10, 14 und 39 basiert $u$. a. auf ideengeschichtlichen und politiktheoretischen Überlegungen zu office, trust, virtue, honour der Amtsinhaber sowie dem popular good in einem System des limited and constitutional government ${ }^{50}$. Dogmatisch klar unterscheidet Hamilton zwischen strafrechtlicher und verfassungsrechtlicher Verantwortung der Inhaber öffentlicher Ämter ${ }^{51}$. Drei Eckpfeiler bestimmen die Autoren für den Bestand und die Entwicklung einer freiheitlichen Republik:

- »ein Menschenbild, in dem die sozialen Tugenden die Korrumpierbarkeit zumindest leicht überwiegen,

47 Art. 34 GG.

48 Hella Mandt, »Responsible Government< und kontinentale Demokratietheorie« in: dies., Politik in der Demokratie. Aufsätze zu ibrer Theorie und Ideengeschichte, BadenBaden 1998, S. 9 ff., S. 11.

49 Wilhelm Hennis, "Amtsgedanke und Demokratiebegriff « in: ders., Die missverstandene Demokratie, Freiburg i. Br. 1973, S. 9 ff., S. 17: »Der >Federalist < ist das Hohe Lied auf die Bedeutung der amtsmäßig verfassten Institutionen. Er geht aus von den Aufgaben und Zwecken einer guten Regierung des Gemeinwesens, weist diese Aufgaben verschiedenen Ämtern zu, gibt ihnen die erforderlichen >powers<, Kompetenzen.«

50 Siehe Dietmar Herz, Die wohlerwogene Republik. Das konstitutionelle Denken des politisch-philosophischen Liberalismus, Paderborn u. a. 1999, S. 151 ff.; Manfred G. Schmidt, Demokratietheorien, Opladen 2000, bes. S. $121 \mathrm{ff}$.

51 Vgl. Alexander Hamilton, 70. Artikel in: Alexander Hamilton / James Madison / John Jay, Die Federalist-Artikel, hg. von Angela Adams und Willi Paul Adams, Paderborn 1994, S. 424 ff. 
- ein Verfassungsgefüge, das Vertrauen und Misstrauen nach den Regeln der politischen Erfahrung und Klugheit und gemäß der jeweiligen historischen Konstellation institutionalisiert und so einander die Waage halten lässt,

- eine verfassungskonforme und gerechte Politik und der wachsame freiheitliche Geist der Gesellschaft « ${ }^{52}$.

Der Trust-Gedanke, der Gedanke der Anvertrautheit eines öffentlichen Amtes, beruht kontraktualistisch auf Willensbeziehungen. Trust ist ursprünglich privatrechtlich gemeint als Auftrag zur uneigennützigen Wahrnehmung fremder Interessen. Aufgrund der lange Zeit in der englischen Rechtstradition fehlenden präzisen Unterscheidung zwischen private und public konnte der Terminus mutieren »zum wichtigsten Begriff zur Bezeichnung der konstitutionellen Bindung des 'government an das 'public good « «53. Bereits bei John Locke ist die Regierung lediglich mit der fiduziarischen Wahrnehmung von Rechten betraut, die in der Substanz beim Volk als Treuhänder verbleiben. Auch Repräsentanten verfügen von Rechts wegen über keine originären, sondern lediglich über derivate Befugnisse im Sinne eines treuhänderischen Rechtsverhältnisses ${ }^{54}$. In den Bestimmungen des Gesellschaftskontraktes und im göttlichen und säkularen Recht findet die Treuhandschaft der Regierung ihre Grenzen. Eine Ausübung von Regierungsgewalt, die nicht gedeckt ist durch Treuhandstellung, stellt einen Vertrauensbruch dar (breach of trust) und bevollmächtigt das Volk zur Rücknahme des trust ${ }^{55}$.

Mit dem Begriff der »Institutionalisierung delegierter Macht« rezipiert Hamilton im Federalist dieses Verständnis der herrschaftlichen Aufgabe als trust $t^{56}$. Und Madison konkretisiert die Ziele einer politischen Verfassung: »erstens, als Herrscher solche Männer gewinnen, die den schärfsten Blick haben, das Gemeinwohl der Gesellschaft zu erkennen und die größte (politische) Tugend, es tatkräftig zu verfolgen«. Zweitens »sind die wirksamsten Vorkehrungen zu treffen, deren Tugendhaftigkeit zu bewahren, solange sie das ihnen übertragene öffentliche Amt in Treuhänderschaft ausüben ${ }^{57}$. Verantwortung und Kontrolle bilden hier ein Gegengewicht zu dem in einer demokratischen Verfassungsordnung erteilten Mandat zum Handeln für die Gesamtheit: »Power and strict accountability for its use are the essential constituents of good government. $\aleph^{58}$ Die Befugnis zu regieren, für andere verbindlich zu ent-

52 Gesine Schwan, »Politik ohne Vertrauen?« in: Peter Haungs (Hg.), Politik obne Vertrauen?, Baden-Baden 1990, S. 9 ff., S. 22.

53 Hubertus Buchstein, »Repräsentation ohne Symbole - Die Repräsentationstheorie des >Federalist< und von Hanna F. Pitkin « in: Gerhard Göhler u. a., Institution - Macht Repräsentation, Baden-Baden 1997, S. 376 ff., S. 392; vgl. auch Benedikt Haller, Repräsentation. Ibr Bedeutungswandel von der hierarchischen Gesellschaft zum demokratischen Verfassungsstaat, Münster 1987, S. 100.

54 Vgl. Peter Saladin, Verantwortung als Staatsprinzip, Bern/Stuttgart 1984, S. 42 f.

55 Vgl. John Locke, Zwei Abhandlungen über die Regierung (1689), hg. und eingeleitet

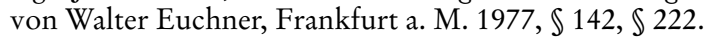

56 Alexander Hamilton, 76. Artikel in: Alexander Hamilton / James Madison / John Jay, aaO. (FN 51), S. 462.

57 James Madison, 57. Artikel in: ebd., S. 346.

58 Woodrow Wilson, Congressional Government, Boston 1985, S. 213 f. 
scheiden, wird als übertragene Vollmacht ausgeübt und ist rechtlich eingegrenzt. »Die Idee des Amtes ist mit unbegrenzter Handlungsfreiheit unvereinbar. Die Befugnis, für andere verbindlich zu entscheiden, hat eine Bestimmung, die nicht zur Disposition des Amtsinhabers steht, sondern ihm vorgegeben ist. Die Bestimmung des Amtes ist das Gemeinwohl. Wer befugt ist, für andere zu entscheiden, muß sich verantworten. Verantwortlichkeit ist möglicherweise das konstitutive Element des Amtes überhaupt. «59

Die mit dem Verantwortlichkeitsprinzip umrissenen strukturell-institutionellen Responsivitäts- und Kontrollmuster im Sinne einer »Organisationstechnik einer funktional differenzierten staatlichen Kompetenzordnung mit Zuständigkeiten, Ämtern und Kontrollinstanzen $\ll^{60}$ werden im Konzept des responsible government konstitutiv verknüpft mit normativen und partizipatorischen Komponenten. In einem Brief aus dem Jahre 1799 an Tench Coxe sieht Thomas Jefferson politische Ämter grundsätzlich als »eine Quelle der Versuchung « und der »Korruption« an. Deshalb müsse man der stets drohenden Arroganz der Macht, dem Ämtermissbrauch und der Petrifikation politischer Herrschaftsverhältnisse mit einer Verfassungsordnung begegnen, welche auf einer Vielzahl von checks and balances grün$\mathrm{de}^{61}$. Allerdings sind - erfahrungsgemäß - rechtliche Vorkehrungen und eine machthemmende Institutionenordnung nicht hinreichend, um Amtsmissbrauch zu verhindern. Es müssen deshalb hinzukommen regierungsfähige, gemeinwohlorientierte Eliten ${ }^{62}$ und unter den Menschen »ein gewisses Maß an Tugend und Ehre (...), das als Basis für solches Vertrauen dienen und es rechtfertigen kann « ${ }^{63}$.

\section{Good Governance und verantwortliche Amtsführung}

Der zentrale Begriff des demokratischen Verfassungsstaates ist nach Wilhelm Hennis nicht die Volkssouveränität, nicht der Wille, sondern das Amt. »Alle verfassungsmäßige Kompetenz ist hier Trust, fiduciary power, Treuhand, anvertraute Aufgabe, Amtsgewalt, gegeben zum Zwecke der Realisierung der Zwecke des Gemeinwesens. $\ll^{64}$ In diesem Sinne haben alle öffentlichen Ämter keine Existenz jen-

59 Peter Graf Kielmansegg, »Die Quadratur des Zirkels« in: Ulrich Matz (Hg.), Aktuelle Herausforderungen der repräsentativen Demokratie, Köln u. a. 1985, S. 9 ff., S. 22.

60 Gerlinde Sommer, aaO. (FN 37), S. 175.

61 Vgl. Thomas Jefferson, Brief an Tench Coxe vom 1. Mai 1794 in: Adrienne Koch / William Peden (Hg.), The Life and Selected Writings of Thomas Jefferson, New York 1954, S. $582 \mathrm{ff}$.

62 Ausführlich Hartmut Wasser, »Zur politischen Ethik Thomas Jeffersons « in: Ingeborg Villinger / Gisela Riescher / Jürgen Rüland (Hg.), aaO. (FN 46), S. 259 ff., S. 260.

63 Alexander Hamilton, 76. Artikel in: Alexander Hamilton / James Madison / John Jay, aaO. (FN 51), S. 462.

64 Wilhelm Hennis, aaO. (FN 49), S. 11; ähnlich Peter Saladin, aaO. (FN 54), S. 48: »Gewaltenteilung als Prinzip wechselseitig - und insgesamt gegenüber dem `Souverän< verantwortlicher Amtsführung von Gemeinwesen (im Bundesstaat), Behörden und Personen ist >umfassende Ordnungsidee (Werner Kägi) des modernen Rechtsstaates (...).« 
seits und außerhalb der Bürgerschaft. Bei öffentlichen Ämtern handelt es sich »gewissermaßen um Kristallisationspunkte verantwortlichen, dem Gemeinwohl verpflichteten Handelns « ${ }^{65}$. Trotz des Siegeszuges des homo oeconomicus in der »Wertewandel-Gesellschaft « und trotz der in den New Public Management-Diskursen vorgetragenen Kritik am tradierten Amtsprinzip als einem (angeblich) retardierenden Element für eine Modernisierung des öffentlichen Sektors messen wir nach wie vor einen Amtsinhaber, ob Abgeordneter, Minister oder Beamter, »an einem uns vorschwebenden Modell des guten, seiner Aufgaben und Pflichten bewussten Inhabers eines solchen Amtes «66.

Um diese Fragestellung kreist $u$. a. die aktuelle Werte- und Ethikdiskussion für die öffentlichen Dienste in den sogenannten NPM-Kernanwender-Staaten (Australien, Großbritannien und Neuseeland) $)^{67}$, den USA und Kanada, also in den Ländern, wo New Public Management-Konzepte relativ früh und konsequent implementiert worden sind. Verantwortlichkeits-, Werte- und Ethikfragen für Politiker und Staatsbedienstete sind in verwaltungswissenschaftlichen Journalen zu einem Modethema geworden ${ }^{68}$. Public Service Acts und Civil Service Codes formulieren ethische Standards und normieren ausdrücklich Werte wie Uneigennützigkeit, persönliche Integrität, Objektivität, Verantwortlichkeit, Transparenz, Aufrichtigkeit und Führungskompetenz für die Staatsbediensteten ${ }^{69}$.

65 Rupert Hoffmann, aaO. (FN 45), S. 130.

66 Wilhelm Hennis, aaO. (FN 49), S. 12.

67 Vgl. Rainer Koch, Auf dem Weg zu einem New Public Service. Zu den Hebelgrößen eines allokativ-effizienten Umbaus öffentlicher Dienste, Beiträge zur Verwaltungswissenschaft, Bd. 35, Universität der Bundeswehr Hamburg, Institut für Verwaltungswissenschaft, Hamburg 2000, S. 2.

68 Siehe u. a. Louis C. Gawthrop, Public Service and Democracy. Ethical Imperatives for the 21 $1^{\text {st }}$ Century, New York/London 1998; Charles Garofalo, Ethics in the Public Service: the moral mind at work, Washington, D. C. 1999; Richard A. Chapman, „Ethics in Public Service for the New Millenium « in: ders. (Hg.), Ethics in Public Service for the New Millenium, Aldershot u. a. 2000, S. 217 ff. Nach Daniel Janett, »Korruptionsgefährdung und New Public Management in der schweizerischen Bundesverwaltung « in: Albert Hofmeister (Hg.), aaO. (FN 10), S. 193 ff., S. 206 f., benennen die Mitarbeiter/ innen der Schweizer Bundesverwaltung als ein wichtiges Element zur Korruptionsvermeidung »das exemplarische Vorleben ethischer Standards durch die Verwaltungskader und die politischen Verantwortungsträger «.

$69 \mathrm{Vgl}$. Jeffrey Minson, »Ethics in the service of state« in: Dean Mitchell / Barry Hindess (Hg.), Governing Australia: studies in contemporary rationalities of government, Cambridge 1998, S. 47 ff.; Lord Nolan, First Report of the Committee on Standards in Public Life, Cm. 2850-I, London 1995 (HMSO), S. 14, listet sieben "principles of public life « auf: »selflessness, integrity, objectivity, accountability, openness, honesty, leadership«. Zur Situation in der EU siehe Christoph Demmke / Danielle Bossaert, Die Ethik im Öffentlichen Dienst der Mitgliedstaaten der Europäischen Union (Europäisches Institut für öffentliche Verwaltung (NL) in Zusammenarbeit mit dem irischen Ministerium für Finanzen und der irischen Präsidentschaft), Maastricht 2004. 
Angesichts der weltweit steigenden Zahl von Korruptionsfällen skizziert die $\mathrm{OECD}^{70}$ eine »ethische Infrastruktur « für den öffentlichen Dienst und die politischen Entscheider. Die OECD schlägt vor, die drei Ziele der Staatsmodernisierung (nämlich Wirtschaftlichkeit, Effizienz und Effektivität) um ein viertes zu ergänzen: die Ethik politisch-administrativen Handelns. »Public Sector Ethics « wird auch in Verlautbarungen der Weltbank zu einem Schlüsselfaktor für die Beurteilung von Regierungsarbeit und Systemqualität, zu einem Merkmal von »Good Governance «1 . Die Qualität von »Governance « bemisst sich zunächst nicht nach der individuellen Moral von Amtsinhabern. Was von den Amtsträgern im Sinne einer Institutionenethik ${ }^{72}$ gefordert wird, ist die Führung des öffentlichen Amtes gemäß seinem Sinn und im Rahmen der gesetzten Regeln. Bürger, Wähler und Repräsentierte müssen darauf vertrauen können, dass, wer immer ein öffentliches Amt innehat, »sich - bei allem Streit um das Gemeinwohl - prinzipiell auf die im Amt institutionalisierte Gemeinwohlbindung einlässt, das heißt sich als dem Ganzen verantwortlich begreift « ${ }^{73}$.

Anders als in Deutschland ist im anglo-amerikanischen Raum die Idee des Trust eine Präzisierung des Office-Begriffs. John Locke entfaltet in seinen »Zwei Abhandlungen über die Regierung " die Idee der Rechenschaftspflicht von Amtsträgern: "Denn da alle Gewalt, die im Vertrauen auf einen bestimmten Zweck übertragen wird, durch diesen $Z$ weck begrenzt ist, so muß, wenn dieser Zweck vernachlässigt oder ihm entgegen gehandelt wird, dieses Vertranen notwendigerweise verwirkt sein und die Gewalt in die Hände derjenigen zurückfallen, die sie erteilt haben und die sie nun von neuem vergeben können, wie sie es für ihre Sicherheit und ihren Schutz am besten halten. $\ll^{74}$ Und falls die »exekutive Gewalt irgendwo anders als bei einer Person, die zugleich einen Anteil an der Legislative hat«, liegt, »so ist sie der Legislative sichtlich untergeordnet und verantwortlich und kann nach Belieben geändert und abgesetzt werden « $^{75}$.

Das Verständnis der herrschaftlichen Aufgabe als trust, als anvertrautes Amt, ist das Korrelat zum abendländischen Verständnis aller Herrschaft als einer der Ge-

70 OECD/PUMA, Ethics in the Public Service. Current Issues and Practice, Public Management Occasional Papers No. 14, Paris 1996, S. 27; OECD, The fight against bribery and corruption, September 2000, S. 6. Ähnlich: American Society of Public Administration (ASPA), Codes of Ethics. Internetdokument: http://www.aspanet.org/ member/coe.htm.

71 Vgl. World Bank, World Development Report 1997: The State in a Changing World, Washington 1997; World Bank, World Development Report 2002, Building Institutions for Markets, Washington, D. C. 2002. Aus: http://econ.worldbank.org/wdr/WDR2002 (19.04.2002). Weiterführend: Jacques Bourgault, »Implications de la sbonne gouvernance « in: Joan Corkery (Hg.), aaO. (FN 6), S. 173 ff.

72 Hierzu: Bernhard Sutor, Kleine politische Ethik, Opladen 1997, S. 41 ff.

73 Peter Graf Kielmansegg, »Wenn das Gemeinwohl aus dem Blick gerät« in: FAZ, Nr. 32, 8. Februar 2000, S. 3. Zu den »responsibilities of office« siehe J. R. Lucas, Responsibility, Oxford 1993, S. 182-203.

74 John Locke, aaO. (FN 55), XIII. Kapitel, S. 294.

75 Ebd., S. 295. 
rechtigkeit und dem Gemeinwohl verpflichteten Aufgabe: »All persons possessing any portion of power ought to be strongly and awfully impressed with the idea that they act in trust; and that they are account in that trust to the one great master, author and founder of society. «76 Wenn Verantwortung es wesentlich mit Fragen der Zuständigkeit, der Kompetenzen, der damit verbundenen Begründungs- und Rechenschaftspflicht gegenüber anderen zu tun hat, dann sind Vertrauen und Verantwortung für jedes freiheitliche System unverzichtbare Grundelemente ${ }^{77}$.

Weil rechtliche und institutionelle Vorkehrungen gegen Amtsmissbrauch nur begrenzt wirksam sein können, binden die Federalist Papers die Institutionalisierung delegierter Macht (also: des anvertrauten Amtes) an »ein gewisses Maß an Tugendhaftigkeit und Ehre unter den Menschen (...) $\ll^{78}$. $\mathrm{Zu}$ den Grundvoraussetzungen funktionsfähiger demokratischer Ordnungen gehören dann neben einer machthemmenden Institutionenordnung, also den verfassungsrechtlich fundierten checks and balances, auch ein gewisses Maß an bürgerschaftlicher Integrität sowie die spezifische Qualität der Amtsinhaber ${ }^{79}$. Da eine Demokratie, die die Ressource Vertrauen verwirtschaftet, in letzter Konsequenz handlungsunfähig wird, ist die Integrität der Amtsinhaber alles andere als eine Privatangelegenheit: Sie ist »ein öffentliches Gut $\ll^{80}$.

Die Erhaltung von Legitimität und demokratischem Vertrauen verlangt von den Amtsinhabern in der Ausübung des ihnen übertragenen Amtes mehr als »lediglich « Rechtsgehorsam. Dieser ist eine elementare Voraussetzung, "Amtsethos und Stilempfinden müssen hinzutreten ${ }^{81}$. In diesem Sinne beinhaltet die geforderte Integrität der Amtsinhaber über den bewussten Respekt vor dem Gesetz hinaus auch moderate Umgangsformen und einen kultivierten politischen Stil, eben das, was mit Dolf Sternberger als »demokratische Haltung « bezeichnet werden kann. In Stil, Form und Verfahren staatlichen Handelns gewinnt der demokratische Verfassungsstaat »Entscheidungssicherheit, gestärkte Verantwortlichkeit, wirksame Kontrolle, demokratische Legitimation und Erneuerungskraft $\ll^{82}$.

76 Edmund Burke, The Works, Bd. V, London 1801, S. 227 f.

77 Vgl. die grundsätzlichen Überlegungen zum Verhältnis von Politik und Vertrauen bei: Rainer Schmalz-Bruns, »Vertrauen in Vertrauen? Ein konzeptioneller Aufriß des Verhältnisses von Politik und Vertrauen « in: ders. / Reinhard Zintl (Hg.), Politisches Vertrauen. Soziale Grundlagen reflexiver Kooperation, Baden-Baden 2002, S. 9 ff. Zu den Zusammenhängen zwischen Vertrauen, Institutionenvertrauen und der Funktionsfähigkeit moderner Gesellschaften siehe Martin Endress, Vertrauen, Bielefeld 2002.

78 Alexander Hamilton, 76. Artikel in: Alexander Hamilton / James Madison / John Jay, aaO. (FN 51), S. 462.

79 Vgl. Thomas Jefferson, Brief an Tench Coxe, aaO. (FN 61), S. 582 f. Zur Bedeutung der guten politischen Sitten für eine freie Gesellschaft bereits Alexis de Tocqueville, »De la Démocratie en Amérique «, 2. Teil, in: ders., Oeuvres complètes, édition definitive sous la direction de J. P. Mayer, Paris 1951 ff., S. 129 (612).

80 Peter Graf Kielmansegg, aaO. (FN 73), S. 3.

81 Heinrich Oberreuter, aaO. (FN 5), S. 47 ff., S. 54.

82 Paul Kirchhof, »Gesetzgebung braucht Form und Stil« in: FAZ, 4. September 2002, Nr. 205, S. 8. 


\section{Folgerungen}

Fälle von Korruption und Amtsmissbrauch provozieren immer wieder Diskussionen, ob neue und striktere Gesetzesregelungen geboten sind: eine Erweiterung der strafrechtlichen Bestimmungen, die Verschärfung von Sanktionsmöglichkeiten, Zeitgrenzen für politische Ämter, mehr Transparenz für die Einkommensverhältnisse bei Abgeordneten- und Regierungsämtern, mehr Öffentlichkeit, Rotation in Verwaltungsämtern, interne und externe Kontrollsysteme und auch die Erweiterung direkt-demokratischer Elemente. Verbesserte Gesetze, optimierte Sanktionspotenziale und strukturell-institutionelle Reformen sind gewiss Voraussetzungen für die Begrenzung von Machtmissbrauch und Missmanagement im öffentlichen Sektor. Aber seit der Antike haben Verfassungsdenker die fundamentale Bedeutung dessen hervorgehoben, was kein Rechtsinstitut gewährleisten kann: »das Bewusstsein gemeinsamer Lebensgrundsätze und Umgangsformen ${ }^{83}$. Die Geschichte der »Pathologien der Politik« (Carl J. Friederich) veranschaulicht die Risiken, die der Ansicht inhärent sind, dass es für die Lösung politischer Probleme einzig und allein auf die gute Organisation des Staates im Sinne zweckmäßiger Institutionen, nicht aber zugleich auf eine »normative Grammatik « ${ }^{84}$ ankomme.

Nach Georg Simmel ruht die Verständigung, »ohne die es überhaupt keine menschliche Gesellschaft gäbe, auf einer kleinen Zahl allgemein zugegebener wenn auch natürlich nicht abstrakt bewusster - Normen«. Diese bilden »das Minimum dessen, was von allen, die überhaupt miteinander verkehren wollen, anerkannt werden $m u ß \ll^{85}$. Gegen diesen Minimalkonsens verstoßen Amtsinhaber, wenn sie ihre Positionierung für gemeinwohl-abträgliche $Z$ wecke nutzen, statt - im Sinne Walter Bagehots ${ }^{86}$ - ihre geliehene Rolle als Befugte und Leitfiguren hoch zu halten. Gewiss reicht es nicht aus, den Amtsgedanken in der politischen Praxis hochzuhalten, um damit vor Positionsmissbrauch und Korruption zu schützen, wenn die Geldbörse zum edelsten Körperteil in einer Gesellschaft wird. Doch die Politik unterliegt im demokratischen Verfassungsstaat neben informellen Anstandserwartungen zugleich verfassungsrechtlich fixierten Geboten, dem allgemein Besten zu dienen. Die Geltung verbindlicher Direktiven samt Regelgerechtigkeit muss auch in der Gegenwartsgesellschaft als eine Vision wirksam bleiben, die kritisch-korrigierende Daueranstrengungen der Bürgerschaft veranlasst und verlangt. Denn nur so "gibt (es) immerhin ein gewisses Mindestmaß von Schamgefühl und Anstandspflicht, welche auch in der Politik nicht ungestraft verletzt werden $\ll^{87}$. Insoweit

83 Joachim Fest, »Die ungeschriebenen Regeln « in: FAZ, 17. März 2000, Nr. 65, S. 3.

84 Vgl. Wolfgang Kersting, »Theoriekonzeptionen der politischen Philosophie der Gegenwart: Methoden, Probleme, Grenzen« in: Michael Th. Greven / Rainer Schmalz-Bruns (Hg.), Politische Theorie - beute. Ansätze und Perspektiven, Baden-Baden 1999, S. 41 ff., S. 66.

85 Georg Simmel, »Exkurs über die Negativität kollektiver Verhaltensweisen« in: ders., Soziologie. Untersuchungen über die Formen der Vergesellschaftung, Leipzig 1908, S. 476 f.

86 Vgl. Walter Bagehot, The English Constitution, London 1968.

87 Max Weber, »Wahlrecht und Demokratie in Deutschland «(1917) in: ders., Gesammelte Politische Schriften, hg. von Johannes Winckelmann, Tübingen 1988, S. 245 ff., S. 247. 
kann hier das Sittliche nicht allein als eine Obliegenheit, sondern funktionsrational als ein fundamentales Interesse der Gesellschaft verstanden werden.

Die Frage nach den Zusammenhängen zwischen der Wahrnehmung von Amtsverantwortung, der öffentlichen Begründung politischer Entscheidungen und den »Kosten der Unmoral $\ll^{88}$ findet - zumindest themenbezogen - noch zu »wenig Aufmerksamkeit in der modernen Politikwissenschaft, weil diese normativ begründete Problematik sich aus der vorwiegend funktionalistischen Herangehensweise nicht stellt ${ }^{89}$ bzw. eher von marginaler Relevanz zu sein scheint ${ }^{90}$. Doch wenn sich Politikwissenschaft »als eine Wissenschaft von unseren eigenen menschlichen Ordnungen und Unordnungen « versteht, kann sie sich »niemals mit der bloßen Registrierung von Daten begnügen, sie muß es zum Urteil bringen «91.

Sollte inzwischen »die Erosion von Anstand und Moral zu Wohlfahrtsverlusten« führen und sollten »Moral und Anstand knappe Ressourcen «92 geworden sein, dann stellt sich die Frage nach den Zusammenhängen zwischen der Wahrnehmung von Amtsverantwortung, der öffentlichen Begründung politischer Entscheidungen und den »Kosten der Unmoral «93 umso prägnanter. Eine Politikwissenschaft, insofern sie sich zugleich als eine empirische und normative Wissenschaft versteht $t^{94}$, wird die Frage nach der Amtsverantwortung im freiheitlich-demokratischen Verfassungsstaat der Gegenwart in Kenntnis der Restriktionen und Friktionen des politischen Alltags re-thematisieren. Jedenfalls lässt sich der Amtsgedanke nicht - wie systemtheoretisches und funktionalistisches Gedankengut unterstellen mag - auf »eine Aufgabe oder Leistung, die von einer bestimmten Struktur erbracht wird bzw. erbracht werden soll «95, reduzieren. Vielmehr ist das Amt im demokratischen Verfassungsstaat »die Bedingung der Möglichkeit des Verfassungsstaates, der darauf angelegt ist, dass Gesetze herrschen und nicht persönliche Willkür, dass der allgemeine Wille, der sich im demokratischen Gesetz verkörpert, gerecht und wirksam umgesetzt wird«96.

88 Guy Kirsch, »Die Kosten der Unmoral « in: FAZ, 19. Oktober 2002, Nr. 243, S. 13.

89 Julia von Blumenthal, Amtsträger in der Parteiendemokratie, Opladen 2001, S. 41 f.

90 Ausführlich: Anthony H. Birch, The Concepts and Theories of Modern Democracy, London 1993, S. 220, $224 \mathrm{ff}$.

91 Dolf Sternberger, zitiert nach Arnd Morkel, »Dolf Sternberger, der Lehrer und Forscher « in: Dolf Sternberger, Das Wort Politik und der Begriff des Politischen, Trierer Universitätsreden, Bd. 10, Trier 1982, S. 23 ff., S. 27.

92 Guy Kirsch, aaO. (FN 88).

93 Ebd.

94 Vgl. Dolf Sternberger, Der Staat des Aristoteles und der moderne Verfassungsstaat. Auseinandersetzungen mit der Antike, Bamberg 1985, S. 13 f.; Henning Ottmann, »In eigener Sache: Politisches Denken « in Politisches Denken. Jabrbuch 1995/1996, S. 1 ff., S. 4. Bernhard Vogel, »Dolf Sternberger zum 80. Geburtstag « in: Zeitschrift für Politik, 34 (1987), H. 4, S. 372 ff., S. 376, bezeichnet die normative Dimension als »die Substanz des eigentlich Politischen «.

95 Peter Birle / Christoph Wagner, »Vergleichende Politikwissenschaft: Analyse und Vergleich politischer Systeme« in: Manfred Mols / Hans-Joachim Lauth / Christian Wagner (Hg.), Politikwissenschaft: Eine Einfübrung, 2. Aufl. Paderborn 1996, S. 102 ff., S. 111.

96 Josef Isensee, »Beamtentum - Sonderstatus in der Gleichheitsgesellschaft « in: Verantwortung und Leistung, H. 18/Juni 1988, S. 5 ff., S. 18. 


\section{Zusammenfassung}

Die einzelstaatlichen und internationalen Bemühungen zur Bekämpfung von Bestechlichkeit und Korruption wie die publizierten Ethikcodes mit grundlegenden Werten für öffentliche Amtsinhaber/innen dokumentieren auch einen Rest jener tradierten Überzeugung, dass Amtspersonen die Wahrnehmung öffentlicher Aufgaben treuhänderisch und mit einer Gemeinwohlorientierung anvertraut worden ist. In zahlreichen Staaten reformulieren inzwischen Ethikkataloge und Integritätskodexe für öffentlich Bedienstete und politische Amtsinhaber/innen die Zusammenhänge zwischen Korruption und Amtsmissbrauch bzw. Korruptionsvermeidung und Amtsethos. Als Schlüsselprinzipien für verantwortliches Regieren und Verwalten (Good Governance) werden traditionelle Werte genannt wie legale und unparteiische Amtsführung, die Integrität der Amtsinhaber und ein Amtsverständnis im Sinne des Gemeinwohls, ergänzt um »neue« Werte wie Effizienz, Effektivität und Transparenz. Die Entwürfe zur Förderung einer »Ethischen Infrastruktur « und zur Implementierung eines »Ethikmanagements « im öffentlichen Sektor reformulieren Prinzipien verantwortlicher Amtsführung, die z. T. bereits im Konzept des Responsible Government entfaltet wurden: eine machthemmende Institutionenordnung, gemeinwohlorientierte Eliten, eine kritischwachsame Bürgerschaft und das Ziel, das verbleibende Misstrauen in Amtsinhaber durch das Vertrauen in die Verfahren und Institutionen einer repräsentativen Demokratie zu kompensieren.

\section{Summary}

The national and international efforts to fight against bribery and corruption and to establish core public sector values or codes of ethics for (public) officials also express elements of such a traditional way of thinking that public office holders act as trustees and have to perform their official duties with honesty, loyalty, and integrity. In many countries concepts of »Ethics Infrastructure « and »Ethics Management« for public servants and for ministers describe the relationship between corruption and misuse of public offices - or better said - contain guidelines on how to avoid corruption and to establish standards of behaviour, such as impartiality in decisionmaking, and upholding the public trust. As core values of responsible governing and public management (Good Governance) are identified traditional values such as legality, integrity, impartiality, and not using the public office for private gain, complemented by »new « values, such as efficiency and transparency. These ethical standards for public office holders and the concept of Good Governance reemphasise the main principles of a Responsible Government: institutional guidelines to prevent the misuse of power, elites who focus on the public good, a critical and aware citizenship, and the goal to replace the remains of mistrust in public office holders by trust in the institutions and decision making processes of a representative democracy. 